\title{
Saturation Throughput of IEEE 802.11e EDCA Based on Mean Value Analysis
}

\author{
Yuxia Lin and Vincent W.S. Wong \\ Department of Electrical and Computer Engineering \\ The University of British Columbia \\ 2332 Main Mall, Vancouver, BC, Canada V6T 1 Z4 \\ E-mail: \{yuxial, vincentw\}@ece.ubc.ca
}

\begin{abstract}
The IEEE 802.11-based wireless LANs have been widely deployed for local area high-speed data access. The IEEE 802.11e amendment aims at providing QoS provisioning to support real-time multimedia traffic in WLANs. The Enhanced Distributed Channel Access (EDCA) is a QoS extension of the Distributed Coordination Function (DCF) in IEEE 802.11a/b/g. In this paper, we propose an analytical model to evaluate the saturation throughput of the IEEE 802.11e EDCA. Our analytical model is based on the use of mean value analysis. We carry out extensive simulation study to validate the accuracy of the proposed model. Our scheme models accurately the effects of the change of contention window size and AIFS (Arbitration InterFrame Space). Our analytical model is applicable to real-time system tuning and on-line admission control algorithms which require a low computation complexity.
\end{abstract}

\section{INTRODUCTION}

Due to the simplicity in deployment and low cost, the IEEE 802.11-based wireless LANs [1] have become the de facto standard for providing high-speed wireless Internet access to end users. IEEE 802.11 Medium Access Control (MAC) specifies two main functions for random access: the contentionbased mechanism of Distributed Coordination Function (DCF) and the centralized mechanism of Point Coordination Function (PCF). DCF is simple and robust, and thus has been used in most commercial products. However, it provides no traffic differentiation, delay or throughput guarantees. Real-time flows may experience arbitrary delays when the network load is high due to the CSMA/CA mechanism. On the other hand, the PCF mechanism can provide a level of service guarantee by a central scheduling scheme, but it introduces additional complexity and protocol overhead. It also has some problems that may lead to poor QoS performance [2]. As a result, PCF is not widely deployed in the existing WLANs.

Driven by the demand of supporting multimedia services on WLANs (such as voice, video and interactive games), the Task Group E in the IEEE 802.11 committee has defined an extension to the IEEE 802.11 standard which provides QoS features and multimedia support to the existing $802.11 \mathrm{a} / \mathrm{b} / \mathrm{g}$ standards while maintaining backward compatibility. The IEEE 802.11e standard [3] defines a new coordination function called the Hybrid Coordination Function (HCF) which includes a contention-based Enhanced Distributed Channel Access (EDCA) mode and a centralized HCF Controlled Channel Access (HCCA) mode.
Due to the rapid deployment of WLANs based on IEEE 802.11 DCF, throughput analysis of the contention-based DCF media access function has been extensively studied by using analytical or simulation methods. One widely used analytical model is proposed by Bianchi [4], which models the binary exponential backoff scheme of DCF with a two-dimensional Markov chain.

With the popularity of the DCF in IEEE 802.11 networks, EDCA is expected to be the dominating access scheme for IEEE 802.11e networks. As a result, performance evaluations of EDCA function have been conducted by using either simulation or analytical methods. Most of the proposed analytical models extend the Bianchi's Markov chain model to take into account the different Arbitration Inter-Frame Space (AIFS) and the different Contention Window (CW) sizes. However, these extensions are not trivial. The use of multi-dimensional Markov chains and other non-linear equations lead to higher computational complexity. It may not be possible to obtain real-time solutions for these equations. Thus, although some of these models provide good accuracy, the high computational complexity may prevent them from implementing in realtime control functions such as on-line call admission control algorithms in IEEE 802.11e networks.

In this paper, we propose a simple and accurate analytical model to evaluate the saturation throughput for 802.11e EDCA access scheme. We extend the work by Tay and Chua [5] and use the mean value analysis. To the best of our knowledge, this is the first paper that utilizes the mean value analysis approach for saturation throughput evaluation in 802.11e networks. The proposed analytical model is simpler, and yet it maintains good accuracy. Our proposed analytical model is validated by extensive simulations. Results show that our model is accurate under a wide range of conditions (i.e., different number of stations, different contention window sizes and different AIFS values). Our model is applicable to the design of fast on-line algorithms such as system parameter tuning and call admission control in 802.11e networks.

This paper is organized as follows. In Section II, we provide an overview of the new QoS access schemes in IEEE 802.11e, and describe the related work on saturation throughput analysis for 802.11 DCF and 802.11e EDCA. In Section III, we describe our proposed analytical model. Section IV presents the results of the model validation by simulation. Conclusions 
and future work are given in Section V.

\section{BACKGROUND AND RELATED WORK}

\section{A. IEEE 802.11e}

IEEE 802.11e is a direct extension to the existing 802.11 networks that provides QoS features and multimedia support, and at the same time, maintains full backward compatibility. It defines a Hybrid Coordination Function, which includes two access modes: a distributed EDCA mode and a centralized HCCA mode.

EDCA provides a prioritized QoS service by enhancing the contention-based DCF function. Higher layer packets are classified into one of the four Access Categories (ACs) at the MAC layer. Each $\mathrm{AC}$ has its own queue and channel access parameters, which include the Arbitration Inter-frame Space (AIFS), minimum and maximum contention window sizes and Transmission Opportunity (TXOP) limits. The ACs gain different priority for channel access by differentiating these parameters. However, due to its probabilistic nature of channel access, EDCA cannot provide hard QoS guarantees such as strict delay bound.

In order to provide parameterized QoS service, HCCA has been proposed as a centralized polling scheme to allocate guaranteed channel access to Traffic Streams based on their QoS requirements. During a beacon interval, a Hybrid Coordinator which is collocated with the QoS Access Point can access the wireless medium with its higher priority to initiate frame exchange sequences and to allocate TXOPs to itself and other stations, so as to provide limited-duration controlled access phase (CAP) for contention-free transfer of QoS data. The durations of TXOPs are calculated from the traffic specification (TSPEC), such as the mean/peak data rate and delay requirement announced at the beginning of the traffic flow. HCCA can be initiated during the Contention-free Period as well as the Contention Period. It is more flexible than PCF. With a good admission control and scheduling scheme, HCCA is able to provide guaranteed QoS services to network flows. However, compared with EDCA, HCCA still presents much challenge for its actual implementation due to its higher complexity and cost concerns. With DCF based $802.11 \mathrm{a} / \mathrm{b} / \mathrm{g}$ products dominating the market, EDCA is expected to be widely adopted as the multimedia solution to the 802.11-based networks. As a result, we focus on EDCA in this paper.

\section{B. Previous Work in Performance Analysis of DCF and EDCA}

With the rapid deployment of the IEEE 802.11 WLANs, there have been many studies of contention-based DCF medium access function. DCF uses the carrier sense multiple access/collision avoidance (CSMA/CA) scheme with binary exponential backoff. Most of the research work focused on the saturation throughput analysis, which is the maximum load that the system can carry in a saturation condition (i.e., the transmission queue of every station is assumed to be always nonempty). This is a fundamental performance figure as it indicates the limit of the network throughput when the offered traffic load increases. In addition to many simulation studies
[6]-[8], analytical models have been constructed for performance analysis. Among these works, Bianchi's model [4] has attracted lots of attention. It models a single station's backoff process with a two-dimensional Markov chain. Assuming that each transmission experiences identical and independent collision probability, it obtains the stationary probability for a station to transmit in a generic time slot. The saturation throughput is obtained by dividing the expected payload information transmitted in a time slot by the expected length of a time slot. This method provides a relatively accurate representation of DCF's binary exponential backoff process, and achieves good performance prediction.

On the other hand, some parallel works derive the saturation throughput without fully describing the detailed behavior of the binary exponential backoff. Cali et al. [9] model the backoff process with a $p$-persistent model. The backoff interval is sampled from a geometric distribution with parameter $p$. They show that the $p$-persistent IEEE 802.11 model closely approximates the standard protocol performance as long as the average backoff interval is the same. They also derive the optimal $p$ value for maximizing the system capacity.

Tay and Chua [5] further simplify the 802.11 MAC modelling by using a mean value approach. Instead of studying the details of the stochastic process using Markov chain, their model tries to approximate the average value for a system variable wherever possible. This technique provides closed-form expressions for the collision probability and the saturation throughput, thus facilitating the analysis of the dependence of system performance on various parameters. The model validation shows that even though this model omits many system details, it still achieves good accuracy warranting its usefulness.

There has been work reported in the literature for the performance of the EDCA function. Experimental testbeds [10] and numerous simulation tests [2], [11]-[13] have been carried out which show that EDCA is effective in traffic differentiation. Several analytical models have also been proposed for 802.11e EDCA. They extend the above DCF models to include the traffic differentiation features of EDCA. Most of the work extends the DCF Markov chain model proposed by Bianchi. Xiao [14] enlarges the original two-dimensional Markov chain to three-dimensional, and analyzes the effects of the different contention window sizes on throughput, but the AIFS-based priority scheme is not included. Kong et al. [15] use a three-dimensional Markov chain to fully describe the backoff behavior of each AC in each station so that both AIFS and CW are considered. Robinson et al. [16] keep the twodimensional Markov chain, but divide the contention periods into different zones to account for the different collision probabilities during different contention periods. Hui et al. [17] also divide backoff into several sub-periods and constructs a P-consistent CSMA/CA model. Ge and Hou [18] extend Cali's $p$-persistent DCF model to include multiple priority classes, but the mathematical model does not incorporate the AIFS differentiation proposed in IEEE 802.11e standard.

One major obstacle for utilizing the analytical models 
based on multi-dimensional Markov chain analysis is the high computation complexity of these models. Solving a set of non-linear equations by approximation may not be feasible in real-time. To better facilitate system design, such as deriving optimal call admission control or adaptive schemes, we need a mathematical model which is accurate and simple to implement (i.e., solutions can be obtained in real-time). The motivation of our work is to propose a simple and yet accurate analytical model.

We achieve this by extending Tay and Chua's mean value DCF model to EDCA. By following the principle of average value approximation, we construct a model based on evaluating the average collision and transmission probability for each access category with different contention window size and AIFS.

\section{The ANALYTICAL Model}

In our proposed model, we assume that there is a fixed number of stations in the network. Each station has saturated traffic with one access category. Though the following work can be generalized to four ACs as defined in the 802.11e standard, we focus our initial analysis on only two ACs. This avoids excessive formulation and makes the model easier to understand. We assume that there are $N_{1}$ stations with access category $A C_{1}$ traffic, and $N_{2}$ stations with access category $A C_{2}$ traffic. $A C_{1}$ is assumed to be the higher priority traffic. We also assume an ideal channel condition which does not have transmission errors or capture effect. TXOP limit is assumed to be 0 , which allows only one frame exchange each time. The system time is considered to be slotted, and only basic access scheme is considered.

For stochastic process analysis, people usually need to adopt assumptions, such as Markov memory and exponential distribution, to make the problem tractable. Network queueing and multi-access systems are especially complex to analyze; only very simplified and ideal protocols can be well analyzed [19]. In our problem, we formulate several simplifying assumptions in the model development to achieve a good trade-off between simplicity and accuracy. The simulation validation later will show that these approximations are good enough to give meaningful system predictions.

\section{A. Differentiation by Contention Window}

We first study the case when all $A C$ s have the same AIFS value. The traffic priority differentiation is thus only realized through differentiating the contention window size. We assume that a packet for $A C_{i}(i=1,2)$ transmitted in a time slot has a constant collision probability of $p_{i}$. Each unsuccessful transmission will be re-tried until an acknowledgment is received. The number of transmissions experienced by each packet is then a geometric distribution with the probability of success $1-p_{i}$. The backoff timer starts with a value uniformly selected from 0 to $W_{i}$, where $W_{i}$ is the minimum contention window size for $A C_{i}$. The average backoff timer in the first stage is thus $W_{i} / 2$.
Each time a collision occurs, the contention window size will double until it reaches the maximum stage $m_{i}$. The average number of backoff time slots experienced by a packet in $A C_{i}$ can be calculated as:

$$
\begin{aligned}
W_{b i}= & \left(1-p_{i}\right) \frac{W_{i}}{2}+p_{i}\left(1-p_{i}\right) \frac{2 W_{i}}{2}+\cdots \\
& +p_{i}^{m_{i}}\left(1-p_{i}\right) \frac{2^{m_{i}} W_{i}}{2}+p_{i}^{m_{i}+1} \frac{2^{m_{i}} W_{i}}{2} \\
& =\frac{1-p_{i}-p_{i}\left(2 p_{i}\right)^{m_{i}}}{1-2 p_{i}} \frac{W_{i}}{2}, \quad i=1,2
\end{aligned}
$$

If we assume that $A C_{i}$ 's packet has a constant probability of transmission in each idle slot, then the probability for $A C_{i}$ 's packet to transmit in an idle time slot can be approximated as:

$$
\tau_{i}=1 / W_{b i}, \quad i=1,2
$$

The probability that one transmitted packet by $A C_{1}$ will collide is the probability that any of the other $\left(N_{1}-1\right) A C_{1}$ stations or $\mathrm{N}_{2} A C_{2}$ stations also transmits:

$$
p_{1}=1-\left(1-\tau_{1}\right)^{\left(N_{1}-1\right)}\left(1-\tau_{2}\right)^{N_{2}}
$$

The same is true for the collision probability of $A C_{2}$ 's packet:

$$
p_{2}=1-\left(1-\tau_{1}\right)^{N_{1}}\left(1-\tau_{2}\right)^{\left(N_{2}-1\right)}
$$

We can solve for $p_{i}$ and $W_{b i}$ from the above system of equations (1)-(4).

To derive the saturation throughput for each $A C_{i}$, we observe the system in a unit time period. The rate of transmission $r_{x i}$ is defined as the number of transmission attempts by $A C_{i}$ stations during this unit time period. The rate of successful transmission $r_{s i}$ is defined as the number of successful transmissions by $A C_{i}$ stations. Due to the geometrical distribution of the number of transmission attempts per packet, the average number of transmissions per packet is $1 /\left(1-p_{i}\right)$, which equals $r_{x i} / r_{s i}$ :

$$
\frac{1}{1-p_{i}}=\frac{r_{x i}}{r_{s i}}, \quad i=1,2
$$

Each collision may involve multiple stations. To a first degree approximation, we assume that each collision involves only two stations. Thus, the rate of collision $r_{c i}$, which is the number of collisions observed in the system in a unit time period, is given by:

$$
r_{c i}=\frac{r_{x i}-r_{s i}}{2}, \quad i=1,2
$$

For $A C_{i}$, we define $T_{i}$ as the time needed to finish a transmission cycle, which is the renewal period between two successive transmissions. $T_{i}$ includes the frame transmission time, an SIFS, the ACK transmission time, an AIFS and an average number of backoff time slots. We make an approximation to estimate the average number of backoff time slots experienced by each packet transmission. At saturation, all transmissions will be preceded with a mandatory backoff selected from the minimum contention window $W_{i}$. If we omit the dependence between two $A C s$ and only look at $A C_{i}$ stations, when $N_{i}$ stations uniformly choose a backoff counter 
from 0 to $W_{i}$, the separation between any two stations has an average of $W_{i} /\left(N_{i}+1\right)$. The station with the smallest backoff counter may make the first transmission after $W_{i} /\left(N_{i}+1\right)$ slots of waiting. As an approximation, we have:

$T_{i}=T_{\text {frame }}+T_{S I F S}+T_{A C K}+T_{A I F S}+\frac{W_{i}}{N_{i}+1} T_{\text {slot }}$

The time taken by the successful and collision transmissions should sum up to one time unit:

$$
\sum_{i=1}^{2}\left(r_{s i}+r_{c i}\right) T_{i}=1
$$

It is reasonable to assume that the rate of transmission by each $A C_{i}$ is proportional to the number of stations in each $A C_{i}$ and the transmission probability by each $A C_{i}$ :

$$
\frac{r_{x 1}}{N_{1} \tau_{1}}=\frac{r_{x 2}}{N_{2} \tau_{2}}
$$

Equations (5)-(9) give a system of linear equations about $r_{x i}$ and $r_{s i}$ which can be solved in closed form:

$$
\begin{array}{r}
r_{x 1}=K\left[\left(1-p_{1} / 2\right) K T_{1}+\left(1-p_{2} / 2\right) T_{2}\right]^{-1} \\
r_{x 2}=\left[\left(1-p_{1} / 2\right) K T_{1}+\left(1-p_{2} / 2\right) T_{2}\right]^{-1} \\
r_{s 1}=K\left(1-p_{1}\right)\left[\left(1-p_{1} / 2\right) K T_{1}+\left(1-p_{2} / 2\right) T_{2}\right]^{-1} \\
r_{s 2}=\left(1-p_{2}\right)\left[\left(1-p_{1} / 2\right) K T_{1}+\left(1-p_{2} / 2\right) T_{2}\right]^{-1}
\end{array}
$$

where $K=N_{1} \tau_{1} / N_{2} \tau_{2}$

The saturation throughput for $A C_{i}$ can be calculated as:

$$
S_{i}=r_{s i} * T_{p, i}, \quad i=1,2
$$

where $T_{p, i}$ is the time used to transmit the frame payload information for an $A C_{i}$ packet.

\section{B. Differentiation by AIFS}

The previous modelling only considers the effects of the contention window size on the throughput differentiation. IEEE 802.11e standard also defines traffic differentiation by utilizing different AIFS. When we further take into account the different AIFS values for each access category, it is more challenging to design an effective mean value-based mathematical model.

Since we assume that $A C_{1}$ is the higher priority access category, it is reasonable to assume that $A I F S_{1} \leq A I F S_{2}$. By the IEEE 802.11e standard [3], AIFS is calculated as

$$
A I F S_{i}=S I F S+A I F S N_{i} * T_{\text {slot }}, \quad i=1,2
$$

where $T_{\text {slot }}$ is the slot time and AIFSN is an integer with a typical value from 1 to 7 .

For simplicity, in the subsequent equations, we assume that

$$
A I F S N_{2}=A I F S N_{1}+M
$$

where $M$ is a non-negative integer.

The problem with introducing different AIFS values is that it may violate some of the assumptions we used before. During the $\left(A I F S_{2}-A I F S_{1}\right)$ time period, there is less contention for $A C_{1}$ traffic. The collision probability during this period may become lower. This violates the basic assumption that a packet belonging to one $A C$ experiences identical collision probability in any time slot. Robinson et al. [16] solve the problem by dividing the transmission periods into two contention zones and calculating the different collision probabilities in each zone. Kong et al. [15] expand the Markov chain to three-dimensional to give a detailed representation of the priority access scheme.

In order to follow the average value methodology, we assume that the collision probability for each access category is still identical in each slot time. This assumption is reasonable if $M$ is small compared to $C W_{\min }$. In this case, the $M$ time slots during which $A C_{1}$ has a smaller collision probability has less significance when we calculate the average probability. With this approximation, we can calculate the new average collision probability for each access category.

Each time the wireless medium becomes idle, $A C_{2}$ will wait $M$ more time slots before counting down its backoff timer. To incorporate the effects of the difference in $A I F S$ into the framework in the previous subsection, we consider these $M$ extra time slots as some equivalent extra backoff time slots experienced by $A C_{2}$. With this consideration, we re-examine equation (1) to find the new average backoff time slots experienced by each access category's packet. The higher priority $\left(A C_{1}\right)$ packet's average backoff slots $\left(W_{b 1}\right)$ remains the same as expressed in equation (1). The effect of longer $A I F S_{2}$ for $A C_{2}$ is converted into $A C_{2}$ 's equivalently longer backoff time slots $W_{b 2}^{\prime}$.

To calculate the total number of time slots which an $A C_{2}$ packet will experience due to its larger AIFS, we first estimate how many times an $A C_{2}$ packet's backoff timer will be interrupted before counting down to zero. An extra $M$ slots' backoff time occurs every time the backoff timer is frozen due to sensing the busy medium.

The probability that $A C_{2}$ 's backoff will be interrupted due to busy medium can be approximated with $A C_{2}$ 's conditional collision probability $p_{2}$. With an average backoff timer of $W_{2} / 2$ in the first stage, it experiences an average of $p_{2} * W_{2} / 2$ interruptions. For each of these backoff timer interruptions, it incurs an extra backoff time slot of $M p_{2} * W_{2} / 2$ compared to $A C_{1}$. Iteratively, these extra $M p_{2} * W_{2} / 2$ backoff slots may introduce another $p_{2} *\left(M p_{2} * W_{2} / 2\right)$ timer interruptions, which lead to another extra $M p_{2} *\left(M p_{2} * W_{2} / 2\right)=M^{2} p_{2}^{2} * W_{2} / 2$ time slots. As a result, the effect of an extra $M$ AIFS time slots for $A C_{2}$ is approximately equivalent to increasing the average first stage backoff slots of $A C_{2}$ from $W_{2} / 2$ to:

$$
\begin{array}{r}
\frac{W_{2}}{2}+M p_{2} \frac{W_{2}}{2}+M^{2} p_{2}^{2} \frac{W_{2}}{2}+\cdots \\
=\frac{W_{2}}{2}\left(\frac{1}{1-M p_{2}}\right)
\end{array}
$$

The adjusted average backoff time slots for $A C_{2}$ becomes

$$
W_{b 2}^{\prime}=\frac{1-p_{2}-p_{2}\left(2 p_{2}\right)^{m_{2}}}{1-2 p_{2}} \frac{W_{2}}{2}\left(\frac{1}{1-M p_{2}}\right)
$$


Note that the above equation is deduced from our assumption that $M$ (the difference between $A I F S_{1}$ and $A I F S_{2}$ ) is relatively small compared to $C W_{\text {min }}$. Too large an $M$ may well lead to $1-M p_{2}<0$, which will invalidate this equation.

By substituting $W_{b 2}^{\prime}$ as $W_{b 2}$ into the system of equations presented in Section III.A, we can obtain the solution for the saturation throughput prediction from equation (14).

\section{Model Validation by Simulation}

In order to verify the validity of the proposed analytical model, we carry out extensive simulations using the ns-2 simulator [20]. The parameters used in the simulation are summarized in Table I. The network has one access point, $N_{1}$ stations with $A C_{1}$ traffic and $N_{2}$ stations with $A C_{2}$ traffic. All the stations are within transmission range of each other. Only basic access scheme is used. Each AC is backlogged with constant bit rate traffic. The packets have a fixed payload size of 1020 bytes.

\begin{tabular}{|l|c|}
\hline \hline Basic Rate & $1 \mathrm{Mbps}$ \\
\hline Data Rate & $11 \mathrm{Mbps}$ \\
\hline DSSS PLCP Preamble and Header & $192 \mathrm{bits}$ \\
\hline MAC Header & $192 \mathrm{bits}$ \\
\hline FCS(Frame Check Sequence) & $32 \mathrm{bits}$ \\
\hline ACK Frame Size & $112 \mathrm{bits}$ \\
\hline Time Slot & $20 \mu \mathrm{s}$ \\
\hline SIFS & $10 \mu \mathrm{s}$ \\
\hline \hline
\end{tabular}

TABLE I

Simulation Parameters

\section{A. Effect of Contention Window Size Differentiation}

We first examine the effects of different contention window size on the saturation throughput. We set $A I F S N_{1}=$ $A I F S N_{2}=2, C W_{\min 1}=15, m_{1}=2$, and $C W_{\min 2}=$ $31, m_{2}=2$ for simulations in Figure 1 according to recommended default parameter settings in the 802.11e standard [3].

Figure 1 shows the aggregated saturation throughput when we symmetrically increase the number of stations in each $\mathrm{AC}$ from 2 to 20 . The aggregated throughput for each AC and the total network throughput from simulation and the analytical model are compared. As we can see that there is good agreement between the analytical and simulation results. Also, the aggregated saturation throughput decreases with increasing number of stations due to increased collision and backoff in the network.

Figure 2 shows the saturation throughput performance when the number of stations for each $\mathrm{AC}$ is asymmetrical in the network. The number of stations in $A C_{1}$ is fixed at $N_{1}=5$, but the number of stations in $A C_{2}$ increases from 2 to 20 . $C W_{\min 1}=7$ and $C W_{\min 2}=15$ are used. Due to the asymmetry in the number of stations, we draw the figure using the throughput per station which is a more meaningful metric. Good prediction by the analytical model is also observed. We can also see that the per station throughput by the higher priority $A C_{1}$ significantly decreases due to the increased competition from the lower priority traffic, even though its own number of stations remains the same. Thus, some admission

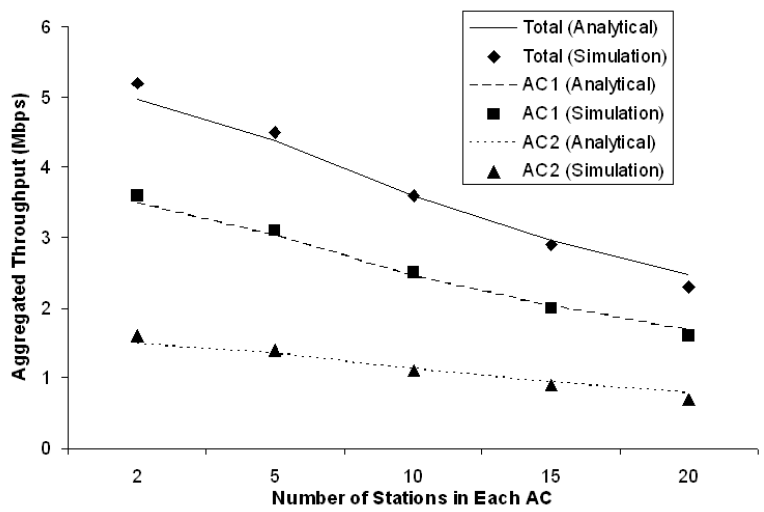

Fig. 1. Throughput performance for symmetrically increasing load

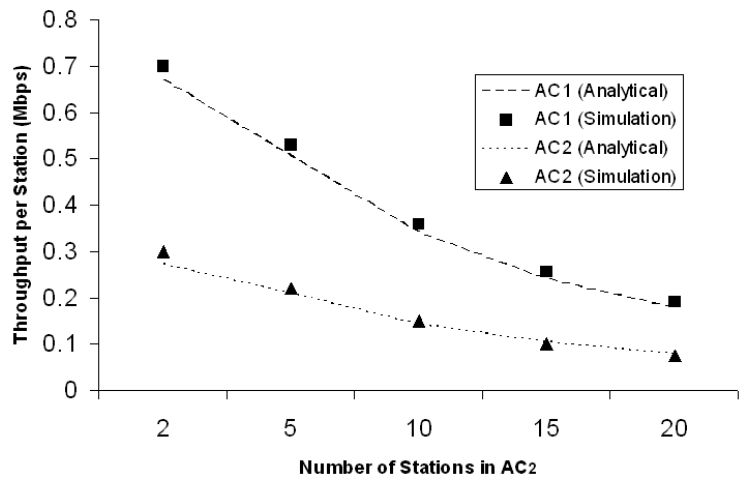

Fig. 2. Throughput performance for asymmetrically increasing load

control mechanism is needed to protect the higher priority traffic's QoS performance from the lower priority traffic.

Figure 3 shows the differentiation effect of contention window size on the saturation throughput by varying the $C W_{\min }$. In this test, $N_{1}=N_{2}=10, m_{1}=m_{2}=2, C W_{\text {min }}$ for $A C_{1}$ is fixed at 7, but the $C W_{\min }$ for $A C_{2}$ increases from 7 to 63 . From the aggregated saturation throughput for each $A C$, we can clearly see the effectiveness of traffic differentiation by the minimum contention window size.

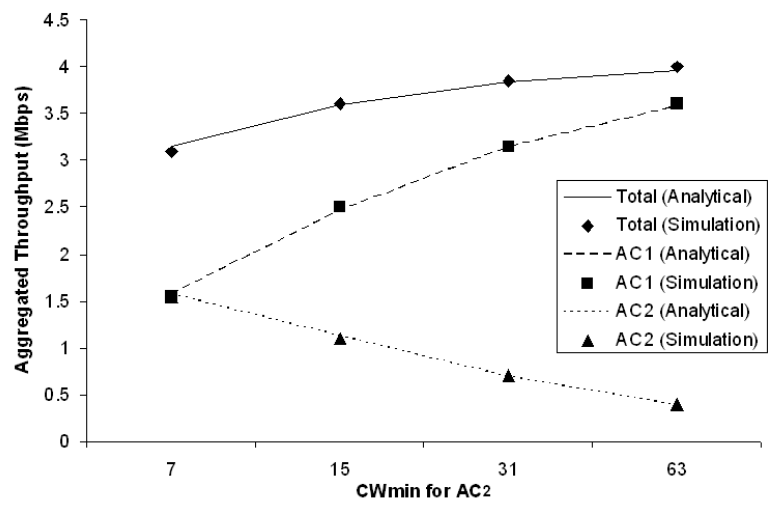

Fig. 3. Differentiation effect of CWmin 


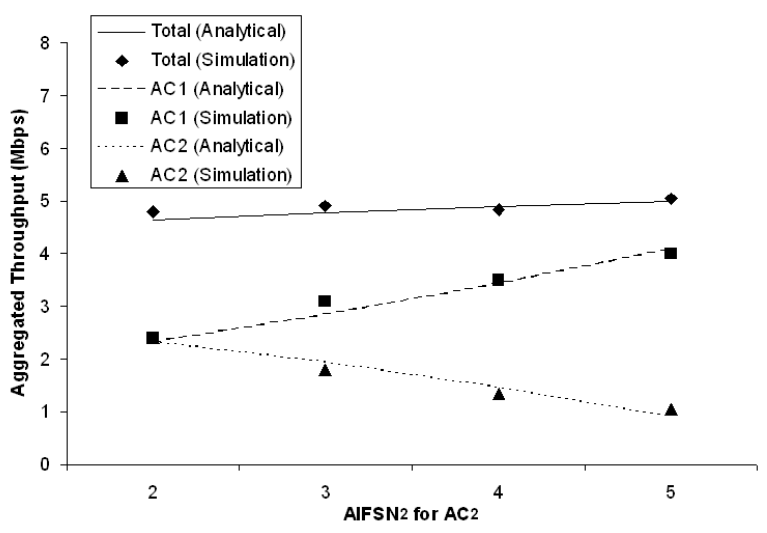

Fig. 4. Differentiation effect of AIFS

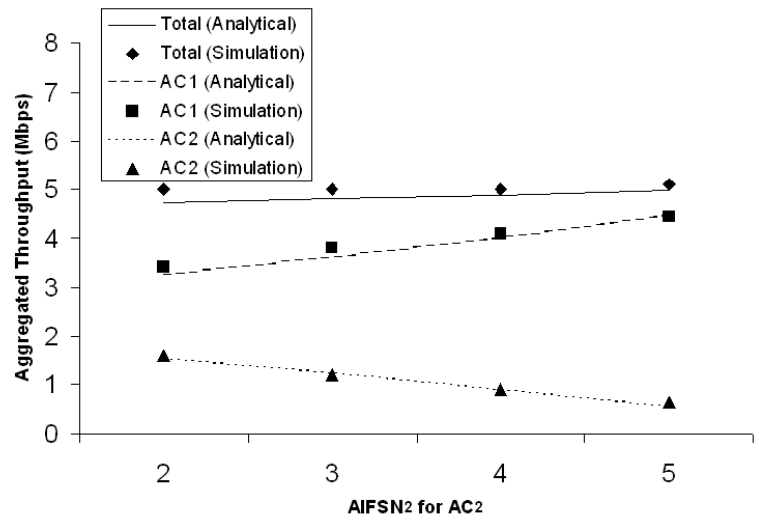

Fig. 5. Differentiation effect of AIFS and CWmin

\section{B. Effect of AIFS Differentiation}

We study the sole effect of AIFS for traffic differentiation by setting the contention window size parameters to be the same for the two access categories and only varying the AIFS value. In this simulation, $N_{1}=N_{2}=5, C W_{\min 1}=C W_{\min 2}=31$, $m_{1}=m_{2}=2, A I F S N_{1}=2 . A I F S N_{2}$ changes from 2 to 5. The results are shown in Figure 4. Comparing Figure 3 and 4, we can see that AIFS has a more dramatic effect on traffic differentiation. Increasing $A C_{2}$ 's AIFS by one has almost the equivalent effect of doubling its $C W_{\min }$ value. But setting AIFS difference to too large a value may lead to traffic starvation for low priority traffic.

Figure 5 shows the combined differentiation effects of $C W_{\min }$ and AIFS on the traffic. In this simulation, $N_{1}=$ $N_{2}=5, C W_{\min 1}=31, C W_{\min 2}=63, m_{1}=m_{2}=2$, $A I F S N_{1}=2$ and $A I F S N_{2}$ increases from 2 to 5 . The results also show good prediction by the analytical model.

\section{CONCLUSiOnS}

In this paper, we proposed a novel analytical model for saturation throughput evaluation of the IEEE 802.11e EDCA access mechanism. The proposed model is based on the use of mean value analysis. The distinct advantage of this model is a lower computational overhead when compared to other multi-dimensional Markov chain approaches. Although there is tradeoff between the model complexity and its accuracy, simulation results show that our model is accurate under a wide range of conditions. Our proposed analytical model is applicable to real-time system tuning and on-line admission control algorithms which require a low computation complexity. For future work, we will continue to improve the accuracy of the model by relaxing more assumptions. We plan to extend the proposed model to determine the average packet delay. In addition, we will also apply the proposed model for call admission control in IEEE 802.11e EDCA networks.

\section{ACKNOWLEDGMENT}

This work is supported by the Natural Sciences and Engineering Research Council of Canada under grant number STPGP 269872-03.

\section{REFERENCES}

[1] IEEE 802.11 WG, "Part 11: Wireless LAN medium access control (MAC) and physical layer (PHY) specifications," 1999.

[2] Q. Ni, "Performance analysis and enhancements for IEEE 802.11e wireless networks," IEEE Network, vol. 19, no. 4, pp. 21-27, July/Aug. 2005.

[3] IEEE 802.11 WG, "IEEE Std 802.11e-2005 (Amendment to IEEE Std 802.11, 1999 Edition (Reaff 2003)," 2005.

[4] G. Bianchi, "Performance analysis of the IEEE 802.11 distributed coordination function," IEEE J. Select. Areas Commun., vol. 18, no. 3, pp. 535-547, Mar. 2000.

[5] Y. Tay and K. Chua, "A capacity analysis for the IEEE 802.11 MAC protocol," Wireless Networks, vol. 7, no. 2, pp. 159-171, Mar. 2001.

[6] G. Bianchi, L. Fratta, and M. Oliveri, "Performance evaluation and enhancement of the CSMA/CA MAC protocol for 802.11 wireless LANs," in Proc. of IEEE PIMRC'96, Taipei, Taiwan, Oct. 1996, pp. 392-396.

[7] B. P. Crow, I. Widjaja, J. G. Kim, and P. T. Sakai, "IEEE 802.11 wireless local area networks," IEEE Commun. Mag., vol. 35, no. 9, pp. 116-126, Sept. 1997.

[8] J. Weinmiller, M. Schlager, A. Festag, and A. Wolisz, "Performance study of access control in wireless LANs-IEEE 802.11 DFWMAC and ETSI RES 10 HIPERLAN," Mobile Networks and Applications, vol. 2, no. 1, pp. 55-67, Mar. 1997.

[9] F. Cali, M. Conti, and E. Gregori, "Dynamic tuning of the IEEE 802.11 protocol to achieve a theoretical throughput limit," IEEE/ACM Trans. Networking, vol. 8, no. 6, pp. 785-799, Dec. 2000.

[10] A. Banchs, A. Azcorra, C. Garcia, and R. Cuevas, "Applications and challenges of the 802.11e EDCA mechanism: An experimental study," IEEE Network, vol. 19, no. 4, pp. 52-58, July/Aug. 2005.

[11] A. Lindgren, A. Almquist, and O. Schelen, "Quality of service schemes for IEEE 802.11 wireless LANs: an evaluation," Mobile Networks and Applications, vol. 8, no. 3, pp. 223-235, June 2003.

[12] S. Choi, J. D. Prado, S. Shankar, and S. Mangold, "IEEE 802.11e contention-based channel access (EDCF) performance evaluation," in Proc. of IEEE ICC, Anchorage, Alaska, May 2003, pp. 1151-1156.

[13] D. He and C. Q. Shen, "Simulation study of IEEE 802.11e EDCF," in Proc. of VTC, Jeju, Korea, Apr. 2003, pp. 685-689.

[14] Y. Xiao, "Performance analysis of IEEE 802.11e EDCF under saturation condition," in Proc. of IEEE ICC, Paris, France, June 2004, pp. 170-174.

[15] Z. Kong, D. H. K. Tsang, and B. Bensaou, "Performance analysis of IEEE 802.11e contention-based channel access," IEEE J. Select. Areas Commun., vol. 22, no. 10, pp. 2095-2106, Dec. 2004.

[16] J. W. Robinson and T. S. Randhawa, "Saturation throughput analysis of IEEE 802.11e enhanced distributed coordination function," IEEE $J$. Select. Areas Commun., vol. 22, no. 5, pp. 917-928, June 2004.

[17] J. Hui and M. Devetsikiotis, "Performance analysis of IEEE 802.11e EDCA by a unified model," in Proc. of IEEE Globecom, Dallas, Texas, Nov. 2004, pp. 754-759.

[18] Y. Ge and J. Hou, "An analytical model for service differentiation in IEEE 802.11," in Proc. of IEEE ICC, Anchorage, Alaska, May 2003, pp. 1157-1162.

[19] D. Bertsekas and R. Gallager, Data networks, 2nd ed. Prentice Hall, 1992.

[20] Ns-2 simulator. [Online]. Available: http://www.isi.edu/nsnam/ns/ 